\title{
Optimization of Multiple Roughness Characteristics for Turning of AISI 1040 Steel under Different Cutting Conditions
}

\section{Tanveer Haque ${ }^{1}$, Shubham Kumar ${ }^{1}$, Devjyoti Upadhaya ${ }^{1}$, Manik Barman ${ }^{1}$ and Arkadeb Mukhopadhyay ${ }^{2,{ }^{*}}$}

${ }^{1}$ Department of Mechanical Engineering, Heritage Institute of Technology, Kolkata - 700107, India

${ }^{2}$ Department of Mechanical Engineering, Jadavpur University, Kolkata - 700032, India

* Corresponding author (Email: arkadebjume@gmail.com)

Keywords: Turning; AISI 1040 steel; Optimization; Grey relational analysis; ANOVA.

\begin{abstract}
The present work aims to optimize multiple roughness characteristics i.e. centre line average, root mean square and mean line peak spacing roughness parameters for AISI 1040 medium carbon steel for turning operation. The turning parameters considered are feed rate, depth of cut and cutting condition and are varied at three different levels. Since the present investigation considers three process parameters at three different levels, the combinations laid down in Taguchi's $\mathrm{L}_{9}$ orthogonal array is employed to carry out the experiments. Grey relational analysis is used for the optimization. Optimal surface roughness is achieved for a depth of cut of $0.4 \mathrm{~mm}$, feed rate of $0.07 \mathrm{~mm} / \mathrm{rev}$ and under water cooled cutting condition. Analysis of variance revealed the highest contribution from feed rate in controlling the surface roughness.
\end{abstract}

\section{Introduction}

Turning is a process of material removal from the outer surface of cylindrical or conical components with the help of a single point turning tool. Turning is one of the most fundamental and most widely used material removal processes in a real manufacturing environment among a number of material removal processes. The quality of manufactured products is determined by its surface quality or surface roughness which is a combination of a number of peaks and valleys or impressions left by the cutting tool on machined surface. The characteristic evidence left on the machined surface in the form of finely spaced micro irregularities by the cutting tool which is measured by different roughness parameters such as amplitude, spacing and hybrid. Surface roughness of cutting process has been studied intensively, mostly through experiments. Davim et al. [1], developed a surface roughness prediction model using artificial neural network (ANN) to investigate the effects of cutting conditions during turning of free machining steel, $9 \mathrm{SMnPb} 28 \mathrm{k}(\mathrm{DIN})$. The $\mathrm{ANN}$ model for centre line average roughness $\left(R_{a}\right)$ and maximum peak to valley height $\left(R_{t}\right)$ was developed with the cutting parameters such as feed rate, cutting speed and depth of cut as process parameters. The experiment was planned as per Taguchi $\mathrm{L}_{27}$ orthogonal array. Jiao et al. [2] used fuzzy neural network (FAN) to model surface roughness in turning operations. A model representing the influences of machining parameters on surface roughness was established and then the model was verified by the use of the results of pilot experiments.

The influence of turning parameters such as cutting speed, feed rate and depth of cut on the quality of surface produced for S456 steel bars was investigated by Nian et al. [3]. Taguchi's optimization technique was employed by them and it was concluded that surface roughness can be improved through this approach instead of using engineering judgement. Abburi and Dixit [4] developed a knowledge based system for the prediction of surface roughness in turning process using neural networks and fuzzy set theory. The performance of the developed knowledge-based system was studied with the experimental data of dry and wet turning of mild steel with HSS and carbide tools. Nalbant et al. [5] used Taguchi method to find the optimal cutting parameters for surface roughness in turning. Orthogonal array (OA), signal-to-noise ratio and analysis of variance (ANOVA) were employed to study the performance characteristics in turning operations of AISI 1030 steel bars using TiN coated tools. Three cutting parameters viz. insert radius, feed rate, and 
depth of cut were optimized. Aslan et al. [6] used OA and ANOVA for the optimization of cutting parameters in turning hardened AISI 4140 steel (63 HRC) with $\mathrm{Al}_{2} \mathrm{O}_{3}+\mathrm{TiCN}$ mixed ceramic tool considering flank wear $\left(V_{b}\right)$ and $R_{a}$ as the responses. Statistical analysis of surface roughness and cutting forces during turning of AISI 52100 bearing steel hardened at $64 \mathrm{HRC}$ with CBN tool has been investigated by Bouacha et al. [7] through the response surface methodology (RSM) and ANOVA. The surface roughness is seen to be highly affected by the feed rate whereas cutting speed has a negative effect. In a research work carried out by Lalwani et al. [8] it was revealed that feed rate is the most significant factor influencing the surface roughness for finish hard turning of MDN250 steel. Sahoo et al. [9] revealed the significance of fractal dimension in determining the surface topography in machining. CNC turning and process optimization of mild steel, aluminium and brass were carried out considering fractal dimension as the response. In another study by Sahoo [10], optimization of surface roughness parameters such as $R_{a}$, root mean square $\left(R_{q}\right)$ and mean line peak spacing $\left(R_{s m}\right)$ was carried out using RSM and genetic algorithm. Taguchi method was used by Asiltürk and Akkuş [11] to obtain optimal parametric settings for CNC hard turning of AISI 4140 using coated carbide cutting tools. They indicated that feed rate significantly controls the $R_{a}$ and average maximum height of the surface profile $\left(R_{z}\right)$. In the same study, it was also revealed that the interaction effects of feed rate-cutting speed and depth of cut-cutting speed is important too. Lin [12] reported the optimization of tool life, cutting force and surface roughness using Taguchi based grey relational analysis considering cutting speed, feed rate and depth of cut as process parameters. Improvement in response variables by the use of aforesaid method was suggested in the same work. $R_{a}$, roughness maximum and roundness for turning of SKD11 (high carbon high chromium alloy tool steel) was optimized by the application of Taguchi's orthogonal array and grey relational analysis [13]. Cutting speed, feed rate, depth of cut and ratio of cutting fluid (synthetic oil in water) were considered as the machining parameters. Depth of cut was found to be the most influential control factor.

The high friction due to surface irregularities in between tool and work piece leads to increase in temperatures, tool wear, and poor surface quality. In order to decrease the friction, cutting fluids are necessary to be applied during machining which not only decreases the friction between tool and work piece but also it increases the surface quality of the machined surface. However, applying cutting fluids during machining has caused problems like high cost, pollution and hazards to operator's health. Cryogenic cooling condition has been reported to produce significant improvement in surface roughness, cutting forces, tool wear, etc. Aggarwal et al. [14] optimized tool life, surface roughness, cutting force and power consumption in CNC turning of AISI P-20 tool steel using liquid nitrogen as coolant. Lower levels of cutting speed, feed, and depth of cut were concluded to be desirable for optimizing the responses. Gupta and Sood [15] obtained optimized turning parameter combination for AISI 4340 steel. The responses considered were $R_{a}$ and specific cutting force $\left(K_{s}\right)$ whereas the design variables considered were cutting speed, feed and different cooling conditions (dry, wet and cryogenic). They concluded cryogenic environment to be suitable for minimizing $R_{a}$ and $K_{s}$. Use of cryogenic cooling was also observed to improve the overall machining performance for turning of AISI 1040 medium carbon steel compared to dry machining [16]. Dhar et al. [17] investigated the effect of cryogenic cooling on the temperature generated at the tool chip interface and it was concluded that a significant improvement occurs in the same in comparison with dry condition. Vegetable based cutting fluids also prove to be a suitable alternative considering health and environmental aspects [18]. The optimal cutting condition for minimum surface roughness and chip thickness and effect of machining parameters using factorial design technique and grey relational analysis was achieved by Abhang and Hameedullah [19]. In the same study, apart from cutting speed, feed rate and tool nose radius, concentration of solid lubricant in SAE 40 base oil was considered as another process parameter. $10 \%$ boric acid in SAE - 40 base oil along with feed rate $0.06 \mathrm{~mm} / \mathrm{rev}$, cutting speed $1,200 \mathrm{rpm}$ and $0.8 \mathrm{~mm}$ tool nose radius gave the best results.

From an exhaustive literature review, it may be concluded that the modeling and optimization of surface roughness has received wide attention by researchers to achieve desired quality of 
finished product. Taguchi's principle along with grey relational analysis, genetic algorithm, etc., has been used for single as well as multi-objective optimization of turning parameters. Optimization of machining process parameters has been carried out by several researchers but considering mainly the $\mathrm{R}_{\mathrm{a}}$ as the response [12, 13]. Though, Sahoo [10] considered multiple roughness parameters, the cutting environment was not varied. Thus, it can be seen that there is a dearth in studies concerning the optimization of multiple roughness characteristics of a turned product as well as evaluate the suitable cutting environment to achieve it. Hence, the present study is directed towards the same. A machined surface can be characterized by several roughness parameters. Therefore, it is desired that an optimum value of the parameters is achieved after machining. An attempt has been made to evaluate the combination of cutting process parameters which will yield minimum $R_{a}, R_{q}$ and $R_{s m}$. In general, $R_{a}$ represents the arithmetic mean value of the departure of the profile from the centre line along a sampling length. $R_{q}$ denotes the standard deviation of the distribution of surface heights. $R_{s m}$ signifies the mean spacing between peaks, with a peak defined relative to the mean line i.e. a peak must cross above the mean line and then back below it. Three controlling factors including the depth of cut, feed rate and the cutting environment are varied at three levels. Experiments are carried out following the combinations laid down in Taguchi's L9 OA. Finally, grey relational analysis and ANOVA is carried out to predict the optimal parametric combination and analyze their contributions in controlling the responses.

\section{Experimental Details}

In the present work, AISI 1040 steel bar specimens are used as work piece for turning and carry out the experiments. Composition of AISI 1040 medium carbon steel (except Fe) is presented in Table 1. The diameter of the bars used is $35 \mathrm{~mm}$ while the length is $150 \mathrm{~mm}$. The turning process is carried out on a centre lathe machine equipped with a three jaw chuck (Make: Panther). The work piece is held in the same and a rotating tail stock is not necessary due to the smaller length of the work piece. The tool post is equipped with a HSS single point tool material. The process parameters considered are the depth of cut (d), feed rate (f) and cutting environment (e). The three cutting environments/conditions are dry, water and commercially available water based emulsion. The sump is located at the bottom and the lubricant is carried to the cutting point by a pump. Response variables considered are $R_{a}, R_{q}$ and $R_{s m}$. The spindle speed is kept fixed at 240rpm. The design variables and their levels (Table 2 ) are selected from literature review and machine constraints.

Table 1. Composition of AISI 1040 steel.

\begin{tabular}{|l|c|c|c|c|c|}
\hline Composition & $\mathbf{C}$ & $\mathbf{S i}$ & $\mathbf{M n}$ & $\mathbf{S}$ & $\mathbf{P}$ \\
\hline Percentage by weight & $0.35-0.45 \%$ & $0.15-0.4 \%$ & $0.6-0.9 \%$ & $0.04 \%$ & $0.05 \%$ \\
\hline
\end{tabular}

Table 2. Process parameters and their levels.

\begin{tabular}{|l|l|l|l|l|}
\hline \multirow{2}{*}{ Design Factors } & \multirow{2}{*}{ Unit } & \multicolumn{3}{|c|}{ Levels } \\
\cline { 3 - 5 } & & $\mathbf{1}$ & \multicolumn{2}{|c|}{$\mathbf{2}$} \\
\hline Depth of cut (d) & $\mathrm{mm}$ & 0.2 & $0.4^{*}$ & 0.6 \\
\hline Feed (f) & $\mathrm{mm} / \mathrm{rev}$ & 0.07 & $0.283^{*}$ & 0.558 \\
\hline Cutting Environment (e) & - & Dry & Water* & Water based emulsion \\
\hline
\end{tabular}

* initial test condition

After the turning operation the specimens are cleaned with acetone especially the ones turned in water and emulsion prior to evaluating the surface roughness. The cleaned specimens are subjected to surface roughness measurements' using a stylus type profilometer (Taylor Hobson, Surtronic $3+$ ). It is equipped with a diamond stylus having a tip radius $5 \mu \mathrm{m}$. Measurements are taken using $0.8 \mathrm{~mm}$ sampling length and $4 \mathrm{~mm}$ traversing length. The present investigation 
considers three process parameters varied at three levels. Accordingly, $\mathrm{L}_{9} \mathrm{OA}$ is selected based on degrees of freedom of the experiment which requires 9 experiments to be carried out. Three readings were taken for $R_{a}, R_{q}$ and $R_{s m}$ and their average is considered for further analysis. Mid level combination of the process parameters is considered as the initial test run. The combinations of the turning parameters and the corresponding responses acquired according to the $\mathrm{L}_{9} \mathrm{OA}$ are enlisted in Table 3.

Table 3. Experimental combinations and the corresponding responses.

\begin{tabular}{|l|l|l|l|l|l|l|}
\hline Exp. No. & $\begin{array}{l}\text { Depth of cut }(\mathbf{d}) \\
\text { 'mm' }\end{array}$ & $\begin{array}{l}\text { Feed rate (f) } \\
\text { 'mm/rev' }\end{array}$ & $\begin{array}{l}\text { Cutting } \\
\text { Env. }(\mathbf{e})\end{array}$ & $\begin{array}{l}\mathbf{R}_{\mathbf{a}} \\
(\boldsymbol{\mu m})\end{array}$ & $\begin{array}{l}\mathbf{R}_{\mathbf{q}} \\
(\boldsymbol{\mu m})\end{array}$ & $\begin{array}{l}\mathbf{R}_{\mathbf{s m}} \\
(\mathbf{m m})\end{array}$ \\
\hline 1 & 0.2 & 0.07 & Dry & 5.09 & 6.03 & 0.18 \\
\hline 2 & 0.2 & 0.283 & Water & 9.26 & 11.67 & 0.23 \\
\hline 3 & 0.2 & 0.558 & Emulsion & 13.80 & 15.83 & 0.50 \\
\hline 4 & 0.4 & 0.07 & Water & 2.96 & 3.70 & 0.10 \\
\hline 5 & 0.4 & 0.283 & Emulsion & 6.91 & 8.32 & 0.23 \\
\hline 6 & 0.4 & 0.558 & Dry & 19.90 & 23.57 & 0.49 \\
\hline 7 & 0.6 & 0.07 & Emulsion & 5.95 & 7.19 & 0.14 \\
\hline 8 & 0.6 & 0.283 & Dry & 10.35 & 12.60 & 0.29 \\
\hline 9 & 0.6 & 0.558 & Water & 8.83 & 11.03 & 0.39 \\
\hline
\end{tabular}

$\mathrm{R}_{\mathrm{a}}=$ Centre line average roughness; $\mathrm{R}_{\mathrm{q}}=$ Root mean square roughness; $\mathrm{R}_{\mathrm{sm}}=$ Mean line peak spacing

\section{Results and Discussions}

Optimization Using Grey Relational Analysis. Grey relational analysis (GRA) is an excellent tool which deals with uncertain and multi-variate system very efficiently and was introduced by Deng [20]. In general, black represents no information whereas white denotes complete information. GRA handles information between black and white i.e. grey. The relationship between turning parameters and the surface roughness parameters are quite complex and uncertain. Hence it can be efficiently modeled using GRA. The analysis using GRA involves determination of absolute value of data difference between sequences and their correlation [21, 22]. The optimization of multiple roughness criteria is carried out using GRA. The responses are converted to a single multiperformance index known as the grey relational grade (GRG). Further analysis and optimization is carried out using the GRG.

The first step in grey relational analysis is normalization. This is done since different responses have different range of values and units. Normalization may be done using higher - the better, lower - the - better or nominal - the - best quality characteristics depending upon the desired goal pertaining to a response [23]. In the present case, all the responses are normalized using lower - the - better criteria since a lower surface roughness is desired. Lower - the - better characteristic may be represented as:

$$
x_{i}^{*}(k)=\frac{\max _{i}(k)-x_{i}(k)}{\max _{i}(k)-\min x_{i}(k)}
$$

where $x_{i}{ }^{*}(k)$ is the value after grey relational generation while min $x_{i}(k)$ and max $x_{i}(k)$ are respectively the smallest and largest values of $x_{i}(k)$ for the $k^{\text {th }}$ response. The normalized values $R_{a}$, $R_{q}$ and $R_{s m}$ of all the nine experiments are given in Table 4. After normalization of data, grey relational coefficients (GRCs) of the three parameters are calculated. A comparison between the 
ideal (best $=1$ ) and the experimental sequences may be obtained from the GRCs. The GRCs are calculated for $R_{a}, R_{q}$ and $R_{s m}$ using the following equation:

$$
\xi_{i}(k)=\frac{\Delta_{\min }+r \Delta_{\max }}{\Delta_{0 i}(k)+r \Delta_{\max }}
$$

where $\Delta_{0 i}=\left\|x_{0}(k)-x_{i}{ }^{*}(k)\right\|=$ difference of the absolute value between $x_{0}(k)$ and $x_{i}{ }^{*}(k), \Delta_{\min }$ and $\Delta_{\max }$ are respectively the minimum and maximum values of the absolute differences $\left(\Delta_{0 i}\right)$ of all comparing sequences. In Eqn. (2) the term $r$ is the distinguishing coefficient which is used to adjust the difference of the relational coefficient, usually $\mathrm{r} \in\{0,1\}$ [21-23]. The value of $r$ controls the effect of $\Delta_{\max }$. Lower the value of $r$, higher is the distinguishing ability. The value of $\mathrm{r}$ for the present analysis is taken to be 0.5 [21-23]. In this study, the GRCs for $R_{a}, R_{q}$ and $R_{s m}$ are calculated using Eqn. 2 and their corresponding values are enlisted in Table 4. The process of normalization and calculation of GRCS constitute the grey relational generation. Finally, the multiple performance index which is known as the GRG is calculated for each experimental run by finding out the average of the GRCs for an experimental run. The GRG for the nine experiments and their order are again presented in Table 4. A higher value of GRG signifies nearer optimum condition.

Table 4. Grey relational generation and grade.

\begin{tabular}{|l|l|l|l|l|l|l|l|c|}
\hline \multirow{2}{*}{$\begin{array}{l}\text { Exp. } \\
\text { No. }\end{array}$} & \multicolumn{3}{|l|}{ Normalized Values } & \multicolumn{2}{l|}{ Grey Relational Coefficients } & \multirow{2}{*}{ Grade } & \multirow{2}{*}{ Order } \\
\cline { 2 - 8 } & $\mathbf{R}_{\mathbf{a}}$ & $\mathbf{R}_{\mathbf{q}}$ & $\mathbf{R}_{\mathbf{s m}}$ & $\mathbf{R}_{\mathbf{a}}$ & $\mathbf{R}_{\mathbf{q}}$ & $\mathbf{R}_{\mathbf{s m}}$ & & \\
\hline 1. & 0.874 & 0.883 & 0.808 & 0.799 & 0.810 & 0.723 & 0.7774 & 2 \\
\hline 2. & 0.628 & 0.599 & 0.684 & 0.574 & 0.555 & 0.613 & 0.5803 & 5 \\
\hline 3. & 0.360 & 0.389 & 0.000 & 0.439 & 0.450 & 0.333 & 0.4074 & 8 \\
\hline 4. & 1.000 & 1.000 & 1.000 & 1.000 & 1.000 & 1.000 & 1.0000 & 1 \\
\hline 5. & 0.767 & 0.767 & 0.684 & 0.682 & 0.683 & 0.613 & 0.6590 & 4 \\
\hline 6. & 0.000 & 0.000 & 0.034 & 0.333 & 0.333 & 0.341 & 0.3359 & 9 \\
\hline 7. & 0.823 & 0.824 & 0.909 & 0.739 & 0.740 & 0.846 & 0.7751 & 3 \\
\hline 8. & 0.564 & 0.552 & 0.530 & 0.534 & 0.528 & 0.515 & 0.5257 & 7 \\
\hline 9. & 0.653 & 0.631 & 0.289 & 0.591 & 0.575 & 0.413 & 0.5264 & 6 \\
\hline
\end{tabular}

$\mathrm{R}_{\mathrm{a}}=$ Centre line average roughness; $\mathrm{R}_{\mathrm{q}}=$ Root mean square roughness; $\mathrm{R}_{\mathrm{sm}}=$ Mean line peak spacing

One of the important features of an orthogonal array is that the effect of each parameter on the GRG can be separated at different levels. For example, if the mean GRG for depth of cut (d) at level 1 is to be calculated, the average of grey relational grade for the first three experiments is calculated. Similar calculations are done for all the three factors at all the three levels and enlisted in Table 5. This is known as response table for the means. The table also consists of rank based on delta values. The delta value is calculated by subtracting the lowest value from the highest in a column. A larger delta value indicates higher significance of the parameter in controlling the response. In the response table (Table 5) it can be seen that feed rate (f) has been assigned a rank 1 which means it is the most significant parameter in controlling the surface roughness followed by cutting environment (e). Depth of cut (d) is observed to be the least significant parameter. The corresponding main effects plot is shown in Fig. 1. From the main effects plot, the combination of parameters for minimum roughness criteria is seen to be $\mathrm{d} 2 \mathrm{f} 1 \mathrm{e} 2$ (depth of cut $=0.4 \mathrm{~mm}$, feed rate $=$ $0.07 \mathrm{~mm} / \mathrm{rev}$ and cutting environment $=$ water $)$. This corresponds to experiment number 4 in the present $\mathrm{L}_{9}$ design matrix. The main effects plot also denotes the significance of a parameter in controlling the response. A higher slope of a plot indicates higher significance. Therefore, a high significance of the feed rate is also observed in Fig. 1 similar to the observation made in Table 5. 
Though, the statistical significance may be obtained from analysis of variance. The interaction plots of the cutting parameters are depicted in Fig. 2. They indicate the importance of the interaction effects of the parameters on the multi-response index i.e. the GRG in this case. Non-parallelism of lines in the interaction plots denotes significant interaction [24, 25]. From Fig. 2(a) it can be seen that the depth of cut and feed rate has some interaction effects. The interaction of depth of cut and cutting environment seems to be highly significant in controlling the surface roughness (Fig. 2(b)). But the lines in Fig. 2(c) are seen to be almost parallel and do not intersect which indicates low contribution from the interaction of feed rate and cutting environment.

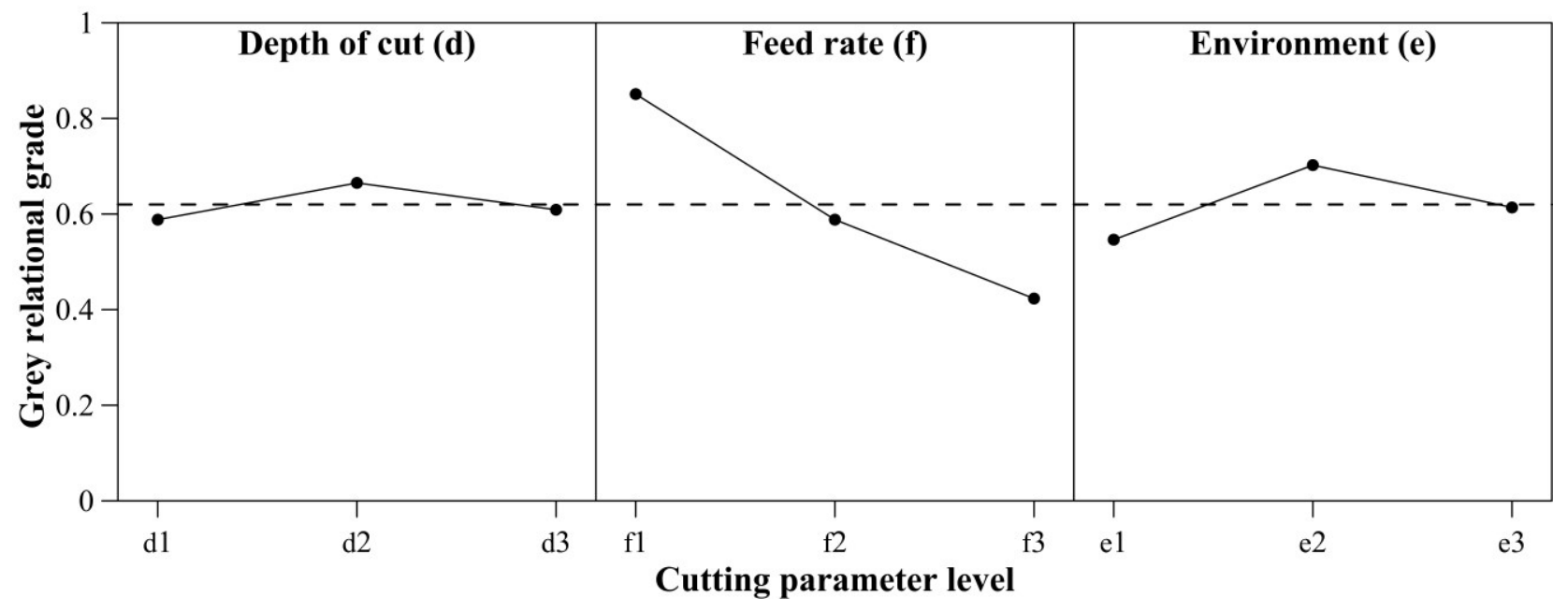

Figure 1. Main effects plot for means of grey relational grade.

Table 5. Response table for means of grey relational grade.

\begin{tabular}{|l|l|l|l|}
\hline Level & Depth of cut (d) & Feed rate (f) & Cutting environment (e) \\
\hline 1 & 0.5883 & 0.8508 & 0.5463 \\
\hline 2 & 0.665 & 0.5883 & 0.7022 \\
\hline 3 & 0.609 & 0.4232 & 0.6138 \\
\hline Delta & 0.0766 & 0.4276 & 0.1559 \\
\hline Rank & 3 & 1 & 2 \\
\hline
\end{tabular}

Average grade $=0.620$

Analysis of variance. The statistical significance of the turning parameters is obtained by carrying out analysis of variance (ANOVA). The ANOVA results for GRG are presented in Table 6 . ANOVA table generally comprises of the corresponding degrees of freedom (DOF) of the factors, sum of squares (SS), mean squares (MS), F-ratio and percentage contribution. The F-ratio is also known as the variance ratio. It is defined as the ratio of regression mean square and the mean square error [21 - 25]. At a particular confidence level, if the calculated F-ratio is greater than the tabulated values for a parameter, then it is considered to cast a significant effect on the response. In the present study, feed rate is predicted to be the most significant cutting parameter at a confidence level of $99 \%$ and with $82.09 \%$ contribution. Similar observations are made by other researchers [7, $8,11]$. The depth of cut and cutting environment contributes $2.77 \%$ and $10.79 \%$ to the surface roughness during the turning process.

Confirmation test. The last step of optimization using GRA is the confirmation test. The confirmation test is carried out to evaluate if there has been any improvement in the GRG compared to the initial test condition. Mid-level combination of the turning parameters is considered to be the initial test run as indicated in Table 2. Moreover, the GRG at the optimal condition is predicted using the following equation: 


$$
\hat{\eta}=\eta_{m}+\sum_{i=1}^{o}\left(\bar{\eta}_{i}-\eta_{m}\right)
$$

where $\eta_{m}$ is the mean grade of all the 9 experiments, $\bar{\eta}_{i}$ is the mean grade at the optimal level for each of the process parameters, and $o$ is the number of main design parameters that significantly affect the surface roughness parameters during the turning process. Table 7 shows the confirmation test results for the present experimental investigation. The predicted GRG is calculated using Eqn. 3. The predicted and experimental grade is in agreement with each other. A very high improvement in the GRG of $58.23 \%$ is obtained which indicates the significance and applicability of the GRA in successfully optimizing the turning process parameters. Moreover, there has been a significant improvement in the roughness parameters. Thus, GRA has been used gainfully in optimizing the turning process and achieve enhanced surface roughness.
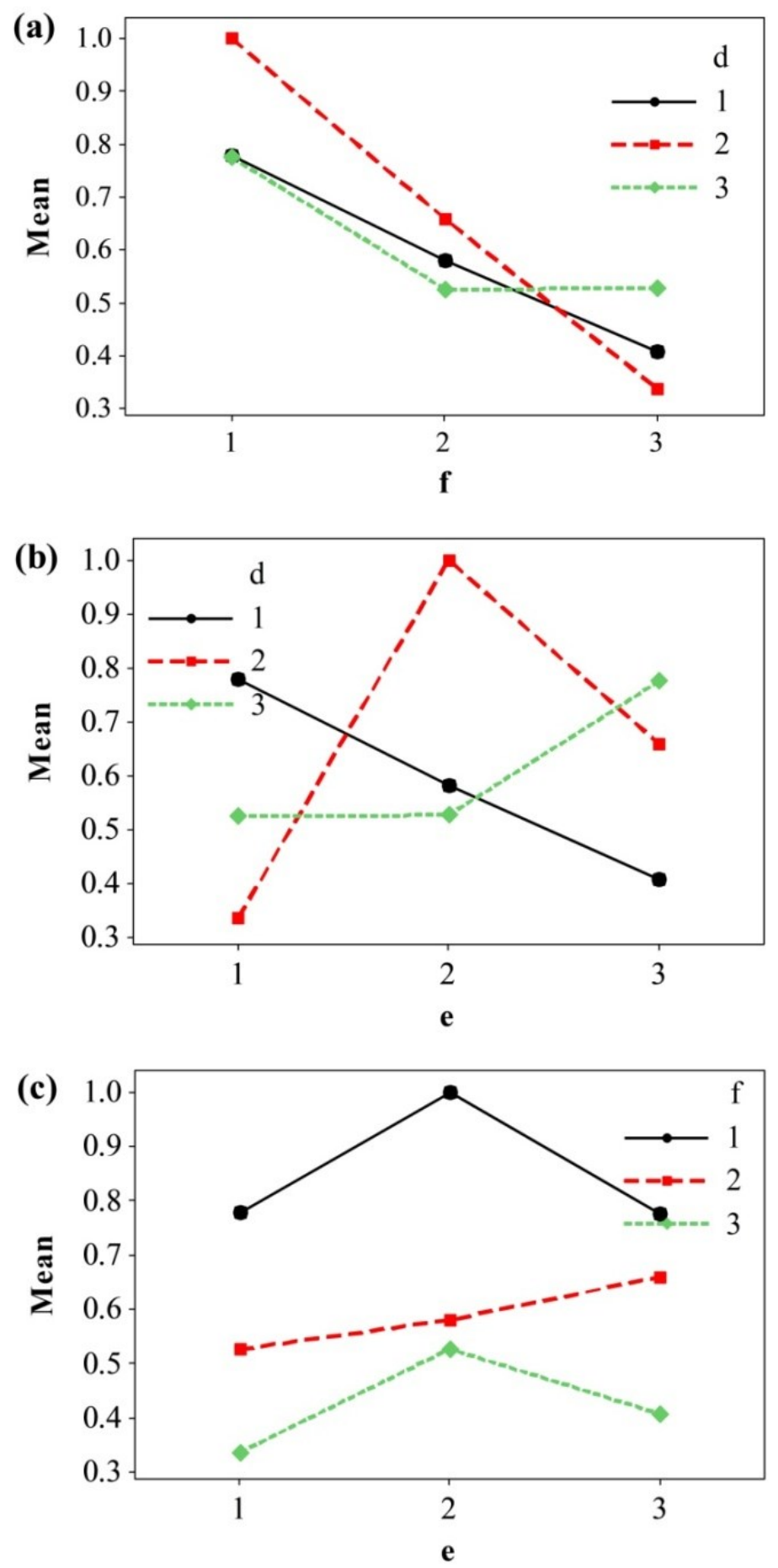

Figure 2. Interaction plots of the cutting parameters (a) d vs. f; (b) d vs. e; and (c) f vs. e. 
Table 6. ANOVA results for turning parameters.

\begin{tabular}{|l|l|l|l|l|l|}
\hline Source & DOF & SS & MS & F-ratio & Contribution (\%) \\
\hline Depth of cut (d) & 2 & 0.0094 & 0.0047 & 0.64 & 2.77 \\
\hline Feed rate (f) & 2 & 0.2790 & 0.1395 & 18.88 & 82.09 \\
\hline Cutting Environment (e) & 2 & 0.0367 & 0.0183 & 2.48 & 10.79 \\
\hline Error & 2 & 0.0148 & 0.0074 & & 4.35 \\
\hline Total & 8 & 0.3399 & & & 100 \\
\hline
\end{tabular}

Table 7. Confirmation test results.

\begin{tabular}{|l|l|l|l|}
\hline \multirow{2}{*}{} & \multirow{2}{*}{ Initial level } & \multicolumn{2}{|l|}{ Optimal parametric level } \\
\cline { 3 - 4 } & & Predicted & Experimental \\
\hline Level & $d 2 f 2 e 2$ & $d 2 f l e 2$ & $d 2 f l e 2$ \\
\hline $\mathrm{R}_{\mathrm{a}}(\mu \mathrm{m})$ & 6.01 & & 2.963 \\
\hline $\mathrm{R}_{\mathrm{q}}(\mu \mathrm{m})$ & 7.17 & & 3.7 \\
\hline $\mathrm{R}_{\mathrm{sm}}(\mathrm{mm})$ & 0.225 & & 0.101 \\
\hline Grade & 0.632 & 0.978 & 1 \\
\hline
\end{tabular}

Improvement of grey relational grade $=0.368(58.23 \%)$

\section{Conclusions}

In the present work an attempt to optimize multiple roughness criteria using grey relational analysis has been made. The process parameters considered are depth of cut, feed rate and cutting environment. Spindle speed has been kept fixed at $240 \mathrm{rpm}$. The operations are carried out on a centre lathe machine. The response variables considered are $R_{a}, R_{q}$ and $R_{s m}$. The optimum combination of parameters for simultaneous minimization of $R_{a}, R_{q}$ and $R_{s m}$ is d2f1e 2 i.e. depth of cut of $0.4 \mathrm{~mm}$, feed of $0.07 \mathrm{~mm} / \mathrm{rev}$ and cutting environment is seen to be water. Feed rate is the most significant factor affecting the surface roughness parameters, followed by cutting environment and lastly by depth of cut. It would be quite interesting to note that the cutting environment that has been found to be suitable for surface roughness is water. In most of the studies it has been seen that mineral oils and soluble water based emulsions provide the best performance to obtain a good surface finish. But in the present case, normal running water is seen to be the most effective. This can be due to the spindle speed chosen which directly affects the cutting speed. The spindle speed chosen in the present case is in the lower range due to which the heat generated is lower. So water acts as an effective coolant. When a multivariate and multi-objective system is considered, the synergistic effects of the process parameters on the response variables is quite complex and is very difficult to predict. GRA is quite effective in such a case. This has been proven in the confirmation test which indicates that the application of GRA has caused a significant reduction of surface roughness parameters considered.

\section{Acknowledgements}

The authors gratefully acknowledge the support provided by Department of Mechanical Engineering of Heritage Institute of Technology and also Jadavpur University for providing surface roughness measurement facilities. 


\section{References}

[1] J.P. Davim, V.N. Gaitonde, S.R. Karnik, Investigations into the effect of cutting conditions on surface roughness in turning of free machining steel by ANN models, J. Mater. Proces. Technol. 205 (2008) 16-23.

[2] Y. Jiao et al., Fuzzy adaptive networks in machining process modeling: surface roughness prediction for turning operations, International Journal of Machine Tools and Manufacture. 44(15) (2004) 1643-1651.

[3] C.Y. Nian, W.H. Yang, Y.S. Tarng, Optimization of turning operations with multiple performance characteristics, J. Mater. Proces. Technol. 95 (1999) 90-96.

[4] N.R. Abburi, U.S. Dixit, A knowledge-based system for the prediction of surface roughness in turning process, Robot. CIM-INT. Manuf. 22 (2006) 363-372.

[5] M. Nalbant, H. Gökkaya, G. Sur, Application of Taguchi method in the optimization of cutting parameters for surface roughness in turning, Mater. Des. 28 (2007) 1379-1385.

[6] E. Aslan, N. Camuşcu, B. Birgören, Design optimization of cutting parameters when turning hardened AISI 4140 steel $(63 \mathrm{HRC})$ with $\mathrm{Al}_{2} \mathrm{O}_{3}+$ TiCN mixed ceramic tool, Mater. Des. 28 (2007) 1618-1622.

[7] K. Bouacha et al., Statistical analysis of surface roughness and cutting forces using response surface methodology in hard turning of AISI 52100 bearing steel with CBN tool, Int. J. Refract. Met. H. 28 (2010) 349-361.

[8] D.I. Lalwani, N.K. Mehta, P.K. Jain, Experimental investigations of cutting parameters influence on cutting forces and surface roughness in finish hard turning of MDN250 steel, J. Mater. Proces. Technol. 206 (2008) 167-179.

[9] P. Sahoo, T.K. Barman, B.C. Routara, Taguchi based fractal dimension modelling and optimization in CNC turning, Advances in Production Engineering \& Management. 3 (2008) 205-217.

[10] P. Sahoo, Optimization of turning parameters for surface roughness using RSM and GA, Advances in Production Engineering \& Management. 6 (2011) 197-208.

[11] İ. Asiltürk, H. Akkuş, Determining the effect of cutting parameters on surface roughness in hard turning using the Taguchi method, Measurement. 44 (2011) 1697-1704.

[12] C.L. Lin, Use of the Taguchi method and grey relational analysis to optimize turning operations with multiple performance characteristics, Materials and Manufacturing Processes. 19 (2004) 209-220.

[13] C.J. Tzeng et al., Optimization of turning operations with multiple performance characteristics using the Taguchi method and Grey relational analysis, J Mater. Proces. Technol. 209 (2009) 2753-2759.

[14] A. Aggarwal et al., Optimization of multiple quality characteristics for CNC turning under cryogenic cutting environment using desirability function, J. Mater. Proces. Technol. 205 (2008) 42-50.

[15] M.K. Gupta, P.K. Sood, Optimizing multi characteristics in machining of AISI 4340 steel using Taguchi’s approach and utility concept, J. Inst. Eng. India Ser. C 97 (2016) 63-69.

[16] M.K. Gupta, G. Singh, P.K. Sood, Experimental investigation of machining AISI 1040 medium carbon steel under cryogenic machining: a comparison with dry machining, J. Inst. Eng. India Ser. C. 96 (2015) 373-379. 
[17] N. R. Dhar, S. Paul, A. B. Chattopadhyay, Role of cryogenic cooling on cutting temperature in turning steel, Transactions of the ASME: Journal of Manufacturing Science and Engineering. 124 (2002) 146-154.

[18] M.H. Cetin et al., Evaluation of vegetable based cutting fluids with extreme pressure and cutting parameters in turning of AISI 304L by Taguchi method, J. Cleaner. Prod. 19 (2011) 2049-2056.

[19] L. B. Abhang, M. Hameedullah, Determination of optimum parameters for multi-performance characteristics in turning by using grey relational analysis, Int. J. Adv. Manuf. Technol. 63 (2012) 13-24.

[20] J.L. Deng, Introduction to Grey system theory, J. Grey. Syst. 1 (1989) 1-24.

[21] A. Mukhopadhyay et al., Tribological Performance Optimization of Electroless Ni-B Coating under Lubricated Condition using Hybrid Grey Fuzzy Logic, J. Inst. Eng. India Ser. D. 97 (2016) 215-231.

[22] A. Mukhopadhyay et al., Optimization of friction and wear properties of electroless Ni-P coatings under lubrication using grey fuzzy logic, J. Inst. Eng. India Ser. D. (2016) 1-14.

[23] A. Mukhopadhyay et al., Application of grey fuzzy logic for the optimization of tribological performance of electroless Ni-B coating, Journal of Manufacturing Technology Research. 7 (2015) 1-24.

[24] A. Mukhopadhyay et al., Wear behavior of electroless Ni-PW coating under lubricated condition-a Taguchi based approach, IOP. Conf. Series: Materials Science and Engineering. 149 (2016) 012004.

[25] S. Duari et al., Optimization of wear performance of electroless Ni-B coating under lubrication, International Journal of Engineering and Technologies. 7 (2016) 94-103. 